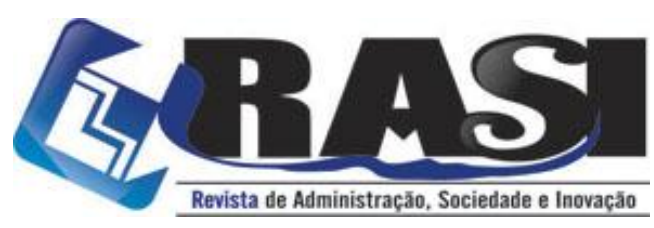

http://www.rasi.uff.br

RASI, Volta Redonda/RJ, v. 3, n. 2, pp. 136-151, jul./dez. 2017

\title{
Los Indicadores De Gestión Ambiental Portuario En España: Una Primera Valoración
}

\author{
Fernando González Laxe (Universidade da Coruña) - laxe@udc.es \\ María Jesús Freire Seoane (Universidade da Coruña) \\ Carlos País Montes (Universidade da Coruña)
}

\section{RESUMEN:}

El trabajo aborda el análisis de los indicadores de desempeño ambiental portuario en España. Se evalúan las distintas fases de su implementación bajo la nueva normativa legal y se formulan los diferentes indicadores e sub-indicadores utilizados, agrupados en las dimensiones económicas, sociales, institucionales y medioambientales. Finalmente, se concluye significando que constituyen una excelente herramienta para el seguimiento de las políticas públicas de sostenibilidad y como "imagen de marca" del sistema portuario y de las propias Autoridades Portuarias.

PALABRAS-CLAVE: sostenibilidad; indicadores; puertos.

\section{Indicadores de gestão ambiental portuária na Espanha: uma primeira avaliação}

\section{RESUMO:}

O trabalho aborda a análise dos indicadores de desempenho ambiental portuário na Espanha. As diferentes fases de sua implementação são avaliadas sob os novos regulamentos legais e os diferentes indicadores e sub-indicadores utilizados formulados, agrupados nas dimensões econômica, social, institucional e ambiental. Finalmente, conclui-se que eles constituem uma excelente ferramenta para monitorar as políticas públicas de sustentabilidade e como uma "imagem de marca" do sistema portuário e das próprias Autoridades portuárias.

PALAVRAS-CHAVE: sustentabilidade; indicadores; portos.

\section{An Approach to Port Environmental Performance in Spain}

\section{ABSTRACT:}

The paper analyzes indicators for port environmental performance in Spain. The different stages involved in their development and implementation are assessed under the new legal framework. Indicators and sub-indicators corresponding to the economic, social, institutional and environmental dimension are also tackled. Finally, these indicators become an excellent tool to monitor public sustainable policies as well as a brand image of port systems and even Port Authorities.

KEYWORDS: sustainability; indicators; ports.

R. Desembargador Ellis Hermydio Figueira, 783, Bloco A, sl. 218, Aterrado.
$27213-415$ - Volta Redonda, RJ - Brasil
www.uff.br




\section{Los Indicadores De Gestión Ambiental Portuario En España: Una Primera Valoración}

\section{Introducción}

La Comisión Europea en su Libro Verde sobre los Puertos (1997) plantea un esquema de tres desafíos. En primer lugar, pretende alcanzar una mayor eficacia en lo tocante a su gestión, permitiendo la movilidad de las mercancías; y, al mismo tiempo, garantizar la posibilidad de elección entre los distintos modos de transporte. En segundo término, apuesta por fomentar la sostenibilidad, limitando las emisiones y los residuos; enfatizando en minimizar el impacto ecológico, utilizando para ello las energías renovables. Y, finalmente, pretende lograr objetivos factibles, es decir, alcanzar el reequilibrio modal; reducir el consumo energético; disminuir las emisiones y los residuos; y reforzar la seguridad.

Dada la amplia heterogeneidad en los sistemas portuarios en Europa, cada país analiza sus rasgos y evalúa las actuaciones de los agentes de la comunidad portuaria desde varias perspectivas. Esto es, actúa desde la estructura del mercado; desde los distintos niveles de la competencia y de la rivalidad; y desde las diferentes posibilidades de regulación pública.

La actividad portuaria ni es ajena a los nuevos paradigmas de la gestión del desarrollo, ni a los nuevos compromisos derivados del desarrollo sostenible (Acciaro et al,2014; Papaefthimiou et al,2017). Para desarrollar una gestión sostenible de un puerto es preciso formular una estrategia que combine los cuatro ámbitos estipulados en el concepto de sostenibilidad: los económicos, los medio-ambientales, los sociales y los institucionales (Peris-Mora et al,2005). Escudriñando dicho análisis, el campo de actuación quedaría delimitado de la siguiente manera. Los objetivos económicos pueden venir señalados por los incrementos del volumen de negocios, los aumentos de los ingresos por concesiones, la reducción del endeudamiento, y la optimización y rentabilización de los activos portuarios. Los retos de la dimensión medio-ambiental se refieren a minimizar los impactos ambientales derivados de la actividad portuaria, reduciendo los accidentes ambientales y mejorando la gestión ambiental. Los objetivos sociales están enmarcados dentro los sistemas de gestión de los recursos humanos, desarrollando equipos humanos comprometidos y logrando un respaldo sostenido y activo de la comunidad del entorno. Finalmente, los desafíos institucionales comprenden los impulsos sobre los cambios legales y normativos para modernizar la forma de desarrollar y operar en el puerto, la reorganización del mercado portuario incorporando aspectos relacionados con la competencia, gestión e inversión privada con el objetivo de mejorar la eficiencia y la capacidad de expansión, modernizar el régimen laboral para mejorar la competitividad, y desarrollar la comunidad portuaria para incrementar la eficiencia operacional y la calidad de los procesos, y expandir la gestión operativa del puerto a la cadena logística para agregar valor e integrar a la comunidad logística local al desarrollo del puerto.

Los objetivos de una Autoridad Portuaria con respecto a la sostenibilidad medioambiental son múltiples y responden a un análisis multidimensional. Un ejemplo de ello se recoge en la Tabla $\mathrm{n}^{\circ} 1$. 
Tabla no 1. Objetivos de una autoridad portuaria con respecto al desarrollo sostenible

\begin{tabular}{|l|l|}
\hline ÁMBITOS & OBJETIVOS \\
\hline Objetivos económicos & $\begin{array}{l}\text { Incrementar el volumen del negocio; aumentar los ingresos por concesiones; } \\
\text { reducir el endeudamiento que garantice la sostenibilidad financiera del puerto; } \\
\text { optimizar y rentabilizar las inversiones de los activos portuarios. }\end{array}$ \\
\hline $\begin{array}{l}\text { Objetivos } \\
\text { ambientales }\end{array}$ & $\begin{array}{l}\text { Fomentar el respeto al medioambiente; minimizar los impactos medio- } \\
\text { ambientales derivados de la actividad portuaria; minimizar los accidentes } \\
\text { ambientales; mejorar la gestión ambiental en el recinto portuario. }\end{array}$ \\
\hline Objetivos sociales & $\begin{array}{l}\text { Desarrollar y modernizar los sistemas de gestión de los recursos humanos; } \\
\text { desarrollar equipos humanos motivados y comprometidos; conseguir un } \\
\text { respaldo activo y sostenido de la comunidad del entorno. }\end{array}$ \\
\hline Objetivos institucionales & $\begin{array}{l}\text { Impulsar cambios legales y normativos para modernizar la forma de desarrollo } \\
\text { y operacional del puerto; reorganización del mercado portuario incorporando } \\
\text { factores como la competencia, la gestión y la inversión privada para lograr } \\
\text { mejorar la eficiencia y la capacidad de expansión; modernizar el régimen } \\
\text { laboral para mejorar su competitividad; desarrollar la comunidad portuaria } \\
\text { para incrementar la eficiencia operacional y la calidad de los procesos; } \\
\text { institucionalizar y optimizar las relaciones puerto-ciudad; expandir la gestión } \\
\text { del puerto a la cadena logística para poder agregar valor; y finalmente, integrar } \\
\text { la comunidad logística local al desarrollo del puerto. }\end{array}$ \\
\hline
\end{tabular}

Fuente: Elaboración propia, a partir de la Guía para la Elaboración de Memorias de Sostenibilidad en el Sistema Portuario Español (2008) y Doerr, O. (2011).

Los objetivos de sostenibilidad quedan enmarcados, por consiguiente, por la creciente demanda de información reclamada por la sociedad; por la mayor transparencia y rigurosidad de la información; por la necesidad de la rendición de cuentas; y por la ejecución de los objetivos del desarrollo sostenible. Ante estas pautas, empieza a ser práctica común la exigencia de elaboración de Memorias de Sostenibilidad y que la propia información económica-financiera proporcionada por las Autoridades Portuarias incluya aspectos relacionados con los factores ambientales y sociales (Kitzman \& Asmus,2006).

El objetivo del trabajo consiste en analizar la puesta en práctica de los indicadores de desempeño ambiental portuario en España, el proceso de selección de los mismos, y la elaboración de un índice sintético que permita efectuar análisis de mejora y de comparabilidad entre las distintas Autoridades Portuarias.

\section{Análisis del sistema de gestión ambiental portuario en España}

\subsection{Rasgos del sistema portuario y análisis de la normativa española}

El sistema portuario español lo componen 46 puertos, integrados en 28 Autoridades Portuarias (AAPP). Es un sistema muy heterogéneo, en donde cohabitan tanto puertos grandes, medianos, como pequeños. Se contabilizan puertos con desiguales niveles de especialización en lo que hace referencia los tráficos de mercancías. Asimismo, existen puertos con diferentes dotaciones infraestructurales y desiguales condiciones relativas a la accesibilidad marítima. Igualmente, se registran puertos con disímiles niveles de conectividad marítima y grados de influencia de los hinterlands próximos a los recintos portuarios. Finalmente, existen puertos con niveles económicos y de promoción comercial muy diferenciados y con resultados muy distintos unos de otros.

Los puertos españoles se encuentran obligados a admitir y a introducir criterios de sostenibilidad en los apartados concernientes a su gestión y a su operativa. Así se ha recogido, 
primeramente, en la ley de Puertos (Ley 33/2010, artículo $3^{\circ}$ ); y, posteriormente, en el Decreto Ley sobre el texto refundido de la Ley de Puertos (D.L, 2/ 2011, artículos 54 y 55).

La Ley 33/2010, novedosa en este aspecto, incluyó, entre otras regulaciones, lo siguiente: a) un esquema de objetivación de la gestión a través de una serie de objetivos e indicadores de sostenibilidad ambiental (artículo 3, apartado 2; que modifica el artículo 39.1 de la Ley 48/2003); b) un mecanismo de información y diálogo con las Autoridades Portuarias (AAPP) a través del desarrollo de Memorias de Sostenibilidad que deben acompañar todos los años a los Planes de Empresa de las citadas AAPP (artículo tercero, apartado 2, que añade un nuevo apartado 4, al articulo 39 de la Ley 48/2003); c) una fuente de financiación estable para medidas de carácter medio-ambiental con cargo al Fondo de Compensación Inter-Portuario (artículo 4.5, b) 3); y d) una llamada de atención en materia de explotación portuaria sobre la necesidad de imponer en los pliegos de prescripciones particulares de los servicios portuarios "las obligaciones de protección medio-ambiental y de contribución a la sostenibilidad" (artículo 62,2,d) así como de valorar en los concursos para el otorgamiento de concesiones, entre otros factores, "las medidas de carácter ambiental y de responsabilidad social corporativa" (artículo tercero, apartado 17).

Las Autoridades Portuarias españolas tienen la obligación de maximizar los beneficios sociales, a través de la optimización de las condiciones de movilidad de las mercancías y personas. Y, para ello, los puertos desempeñan un papel muy relevante en varios planos simultáneos: en primer lugar, en lo referente a los principios de cautela y de acción preventiva; en segundo lugar, en lo que concierne al principio de corrección de los impactos sobre el medio ambiente; y, por último, en lo que atañe al principio "del que contamina, paga".

Estos objetivos se complementan con las siguientes acciones: a) la gestión económica se debe realizar en un marco de desarrollo sostenible, que vele por la protección y la conservación ambiental; b) deben contemplarse acciones que promuevan la integración de los puertos en las ciudades del entorno; y c) se debe fomentar tanto el desarrollo de la intermodalidad como el posicionamiento internacional dentro de las cadenas de suministro global. Todo este armazón estratégico está enmarcado con las políticas de transparencia, con las decisiones de acceso a la información en materia ambiental, y con el desarrollo e implementación de una política de participación pública en la toma de las decisiones relacionadas con el medio ambiente.

El Decreto Ley 2/2011, que refunde los anteriores textos normativos portuarios en un solo texto, pone de manifiesto que, sobre dicha base de instrumentos de gestión, las AAPP han de facilitar y permitir su evaluación y difusión. Es decir, las AAPP han de garantizar la fluidez en la comunicación, ya sean los datos referidos a la información medioambiental, a los resultados vinculados a la eco-eficiencia de la gestión, a la implantación de procesos limpios que reduzcan el impacto ambiental, como a aquellos otros que conduzcan a ahorrar en recursos naturales.

El mencionado texto normativo abarca varias actuaciones tendentes a las mejoras medio-ambientales, como las exigencias a la hora de determinar acciones, ya sea en lo concerniente a la construcción o ampliación de puertos como en las condiciones que regulan los servicios portuarios. Un esquema de lo contemplado en el Decreto Ley. 2/2011 lo exponemos, de manera sintética, en la tabla número 2. 
Tabla $\mathbf{n}^{\mathbf{0}}$ 2.- Bases de la gestión portuaria española en lo referente al desempeño medioambiental, establecido en el Decreto Ley 2/2011.

\begin{tabular}{|c|c|c|}
\hline ÁMBITO & INSTRUMENTO & CONTENIDO \\
\hline Planificación & $\begin{array}{l}\text { Plan Director de } \\
\text { Infraestructuras } \\
\left(\operatorname{art}^{\circ} 54\right) \text {. } \\
\text { Plan de Empresa de la } \\
\text { Autoridad Portuaria (art } \\
\text { 55). }\end{array}$ & $\begin{array}{l}\text { Exige la presentación de una memoria ambiental que debe } \\
\left.\text { ser sometida a evaluación ambiental estratégica ( } \operatorname{art}^{\circ} 54\right) \text {. } \\
\text { Exige la presentación de objetivos e indicadores de } \\
\text { sostenibilidad ambiental del puerto y la evaluación del } \\
\text { impacto ambiental en aquellas actuaciones de de } \\
\left.\text { programas de inversiones públicas (art }{ }^{\circ} 55.1\right) \text {. } \\
\text { Debe acompañar una Memoria de Sostenibilidad acorde } \\
\text { con la metodología aportada por Puertos del Estado (art } \\
\text { 55.4). }\end{array}$ \\
\hline Obras públicas & $\begin{array}{l}\text { Construcción, } \\
\text { ampliación o } \\
\text { modificación del puerto. } \\
\text { Obras de dragado. }\end{array}$ & $\begin{array}{l}\text { Afecta tanto a los proyectos de construcción como de } \\
\text { ampliación o modificación de los puertos, a los que se } \\
\text { exige declaración de impacto ambiental (art } 57.2 \text { y } \text { art }^{\circ} \\
58.1) \text {. } \\
\text { Los dragados han de someterse a evaluación de impacto } \\
\left.\text { ambiental (art }{ }^{\circ} 64\right) \text {. }\end{array}$ \\
\hline Dominio público & $\begin{array}{lr}\text { Exigencias } & \text { de } \\
\text { condiciones } & \text { de } \\
\text { protección del } & \text { medio- } \\
\text { ambiente. } & \end{array}$ & $\begin{array}{l}\text { Afecta a las autorizaciones }\left(\operatorname{art}^{\circ} 80 . e\right) \\
\text { Afecta a las concesiones }\left(\operatorname{art}^{\circ} 87 . e\right)\end{array}$ \\
\hline $\begin{array}{l}\text { Prestación } \\
\text { servicios }\end{array}$ & $\begin{array}{l}\text { Pliego de condiciones de } \\
\text { los servicios portuarios. } \\
\text { Licencias de prestación } \\
\text { de servicios portuarios. } \\
\text { Servicios de recepción } \\
\text { de desechos. } \\
\text { Prestación de servicios } \\
\text { comerciales y de otras } \\
\text { actividades }\end{array}$ & $\begin{array}{l}\text { Regula tanto las condiciones ambientales de la prestación } \\
\text { de servicios como la obligación referida a la protección del } \\
\left.\text { medio-ambiente ( } \operatorname{art}^{\mathrm{o}} 113,4, \mathrm{~d}, \mathrm{e}\right) \text {. } \\
\text { Regula la obligación de protección del medio-ambiente } \\
\left.\text { (art }^{\mathrm{o}} 117,1 . \mathrm{h}\right) \text {. } \\
\text { Regula la organización de las empresas encargadas de la } \\
\left.\text { gestión (art }{ }^{\circ} 132.5\right) \text {. } \\
\text { Determina las condiciones de las empresas y las garantías } \\
\text { que deben cubrir los posibles riegos medio-ambientales } \\
\left(\operatorname{art}{ }^{\mathrm{o}} 139.5, \mathrm{c}, \mathrm{d}\right) \text {. }\end{array}$ \\
\hline Bonificaciones & & $\begin{array}{l}\text { Referidas al buque, por aplicación de Buenas Prácticas en } \\
\text { el medio ambiente. } \\
\text { A los titulares de licencias y concesiones de manipulación } \\
\text { de mercancías o de una terminal de manipulación. } \\
\text { A los titulares de otras actividades (astilleros, servicios } \\
\text { náutico-deportivos, pesca,..) }\end{array}$ \\
\hline $\begin{array}{l}\text { Contaminación } \\
\text { residuos }\end{array}$ & $\begin{array}{l}\text { Prevención y lucha } \\
\text { contra la contaminación } \\
\left(\operatorname{art}^{\circ} 62\right) \text {. } \\
\text { Recepción de desechos y } \\
\text { residuos procedentes de } \\
\text { los buques }\left(\operatorname{art}^{\circ} 63\right) \text {. }\end{array}$ & $\begin{array}{l}\text { Las Autoridades Portuarias colaborarán con las } \\
\text { Administraciones competentes en la prevención y control } \\
\text { de la emergencia por contaminación accidental en las } \\
\text { zonas de servicios. } \\
\text { Las Autoridades Portuarias elaborarán, cada tres años, un } \\
\text { Plan de Recepción de residuos que contempla los desechos } \\
\text { generados por los buques y los residuos de carga. }\end{array}$ \\
\hline $\begin{array}{l}\text { Condiciones } \\
\text { generales }\end{array}$ & $\begin{array}{l}\text { Suscribir } \text { Convenios de } \\
\text { Buenas Prácticas. } \\
\text { Poseer sistemas de } \\
\text { gestión ambiental } \\
\text { certificadas }\end{array}$ & \\
\hline
\end{tabular}

Fuente: Elaboración propia.

Los Memorias de Sostenibilidad Portuaria han de servir, en primer término, para formalizar los objetivos de comunicación externa; o sea, la capacidad de informar de modo 
transparente de las estrategias, de los compromisos y de los progresos de las AAPP en relación a los intereses del entorno con el que se relacionan; y, en segundo lugar, a los objetivos de gestión interna, en la medida que la mencionada Memoria de Sostenibilidad constituye una herramienta de gestión que apoya el desarrollo y aprobación de los planes de empresas, a través de un diagnóstico global de los riesgos y oportunidades ligados a los aspectos institucionales, económicos, sociales y medio-ambientales.

\subsection{Orígenes de la normativa referida a la sostenibilidad ambiental portuaria y los principios incluidos en la misma}

La obligatoriedad de hacer público el dato relativo a la sostenibilidad portuaria tiene su origen en 2008, cuando el Organismo Público Puertos del Estado elaboró, junto a dos Autoridades Portuarias, la de Valencia y A Coruña, una Guía para la elaboración de memorias de sostenibilidad en el sistema portuario español ${ }^{1}$. Dicha propuesta estableció tres dimensiones de análisis: la económica, la social, y la medio-ambiental. Definía los objetivos de cada dimensión y recogía los indicadores básicos para la elaboración de la tabla de indicadores. Más tarde, en vísperas de la tramitación de la modificación de la Ley de Puertos 33/2010, una parte del equipo que elaboró la anterior Guía, propuso una Medición de la Sostenibilidad en el sistema portuario español, titulada "Una propuesta metodológica a través de indicadores sintéticos de desarrollo sostenible" ${ }^{\text {. }}$. Se basaba en abordar los principales desafíos de la gestión sostenible de los puertos a partir de una información que fuera objetiva y confiable sobre el desempeño de las Autoridades Portuarias, permitiendo efectuar un benchmarking de gestión sostenible entre puertos, para determinar las mejores prácticas y poder comparar el desempeño de una AAPP frente a otras industrias similares. Finalmente, Puertos del Estado, en diciembre del 2010, presentó su propuesta final ${ }^{3}$, con su metodología propia, en donde se contemplan los objetivos, avances y contenidos de las cuatro dimensiones. A partir de la misma, se elaboran tanto la Memoria de Sostenibilidad del Organismo Público, como las propias Memorias de las AAPP. Sin embargo, no todas las AAPP presentan las mencionadas Memorias de Sostenibilidad; y no todas las que lo presentan lo efectúan siguiendo la Guía y los objetivos aprobados en la Ley.

La normativa española contempla en su apuesta de sostenibilidad portuaria cuatro dimensiones de análisis. Son las siguientes:

a) Dimensión institucional, en donde se han de describir los principales logros y desafíos de la sostenibilidad medioambiental en lo que se refriere a los modelos de gestión, de identificación y de participación de los grupos de interés, infraestructuras, viabilidad financiera, calidad en la prestación de los servicios y en la innovación del sistema portuario.

b) Dimensión económica, aquella que proporciona información suficiente para evaluar la rentabilidad y viabilidad económica. Comprende los indicadores referidos a la

\footnotetext{
${ }^{1}$ FEPORTS (2008). Guía para la elaboración de Memorias de Sostenibilidad en el sistema portuario español. Madrid

${ }^{2}$ González-Laxe, F., Guerra, A., Martín-Palmero, F., Novoa, J., Otero, C., Penela, J. presentada en la XII Reunión de Economía Mundial. Santiago de Compostela, mayo 2010.

${ }^{3}$ Puertos del Estado, Propuesta de Metodología para la elaboración de Memorias de Sostenibilidad de las Autoridades Portuarias. Madrid, abril, 2011
} 
situación económica-financiera y la información sobre la estructura de las inversiones y los indicadores de rendimientos y productividad.

c) Dimensión social, que basa sus actuaciones en las políticas de recursos humanos; y por lo tanto incluye la estructura de la plantilla, las acciones de formación desarrolladas, los planes de igualdad, y los esfuerzos realizados en materia de seguridad y salud.

d) Dimensión ambiental, en donde se recogen las medidas y los impactos de las acciones llevadas a cabo para la mejora de la gestión ambiental, la calidad de los servicios prestados, y los sistemas de recepción y gestión de las quejas ambientales.

Dichas dimensiones contemplan un triple efecto recíproco. El primero hace referencia a la influencia de la actividad portuaria con el entorno económico, social y ambiental con la que se relacionan. El segundo hace mención al efecto de sus actividades con su propia estructura. Y el tercero, permite que se puedan analizar los efectos de sus funciones y actividades en sus dimensiones económica, social, medio-ambiental e institucional, buscando un desarrollo equilibrado de estas cuatro dimensiones.

Los principios de la sostenibilidad ambiental recogidos en la normativa española se ven reflejados en siete aspectos concretos. En primer lugar, en el campo de la auto-suficiencia económica. Se subraya la no-dependencia de los presupuestos estatales y el cumplimiento de los niveles de auto-suficiencia económica, así como el establecimiento de una rentabilidad minima para todo el sistema portuario (2,5\% anual). Incluye, asimismo, la progresiva participación privada, estableciendo incentivos para su captación. Y, al mismo tiempo, apuesta por la flexibilidad en la aplicación de las tasas, con objeto de adaptarse a la realidad económica, optimizando sus factores de competividad en relación con las redes de comercio, logística y transporte.

En segundo lugar, en el campo de la competitividad, se otorga a las AAPP un liderazgo en el desarrollo y la promoción comercial del puerto. Se obliga, por tanto, a una mayor transparencia en la fijación de las tasas; en lo referente a la gestión de los servicios portuarios y del dominio público-privado; y se garantiza la libre competencia, por medio de regular el libre acceso a las empresas que desean prestar servicios en el puerto.

El tercer principio está relacionado con el campo de la gestión sostenible. Se definen los planes directores, valorando la capacidad de las instalaciones y su grado de utilización; acompañando a dichos planes de las memorias de sostenibilidad y las condiciones de los servicios; así como los pliegos reguladores de los mencionados servicios en donde se deben incluir los criterios de la sostenibilidad exigida.

El cuarto principio determina la apuesta por el campo de la movilidad sostenible de mercancías, a través del fomento del transporte marítimo de corta distancia y las autopistas del mar, el impulso al ferrocarril y la intermodalidad; y de la mejora de los accesos a las instalaciones y recintos portuarios.

En quinto lugar, los principios de la gestión portuaria hacen referencia a la calidad de los servicios. En este sentido, se fijan los impulsos para la mejora de los rendimientos y calidad de los servicios. Se crea el Observatorio de los Servicios Portuarios, con el objetivo de proporcionar una medida de los niveles de competividad y calidad de los servicios prestados por los puertos españoles y extranjeros.

En sexto lugar, en los objetivos de los compromisos portuarios se abordan los asuntos relacionados con la seguridad. Se incluyen en esta materia, tanto el papel coordinador de la 
AAPP en los conceptos de seguridad y protección; como el rol de coordinador y colaborador con otras administraciones públicas. Por último, se enfatiza en la mejora de la señalización marítima y de navegación.

Finalmente, en lo concerniente al campo del medio-ambiente, se subraya el fomento de las nuevas prácticas ambientales y sus incentivos para que se cumplan convenios con las AAPP, en lo que se refieren a la mejora de la eficiencia ambiental de los medios materiales; las condiciones ambientales en los servicios y en las concesiones; y en los servicios MARPOL.

En suma, la normativa española de puertos apuesta de manera clara sobre la sostenibilidad ambiental. Y, atendiendo a sus postulados, cifra objetivos claros y nítidos para su implementación.

\section{Aplicación del sistema de gestión ambiental portuario en España. Definición de los indicadores y las variables}

La gestión del sistema de puertos incluye, en las últimas normativas, incuestionables elementos referidos al ámbito empresarial. Sus continuas adaptaciones y las memorias de sostenibilidad también hacen referencia a dichos parámetros. Se preveía que dichas consideraciones podrían convertirse en elementos eficaces para la gestión económica y sostenible del sistema portuario. Incluso se llegó a afirmar que "el modelo de gestión portuaria considera como principal variable de desarrollo el mencionado factor económico" (Giner et al., 2011).

Las pautas seguidas en la legislación española no hacen más que corroborar esa doble apuesta: la económica y la medio-ambiental. La aplicación de indicadores medio-ambientales portuario se inicia en Valencia, en 2006, con el proyecto MESOSPORT, que daría como último fruto una Guía para la elaboración de memorias de sostenibilidad en el Sistema Portuario Español. Dicha Guía, resultado de dicho proyecto, sigue las recomendaciones del GRI y su filosofía. Como output final de la misma ofrece una batería de indicadores (9 en la dimensión económica, 17 en la ambiental y 25 en la social), que prosiguen, además, la pauta de los definidos en el Cuadro de Mando Integral del Sistema Portuario Español (Anexo 1). A partir de la elaboración de dicha Guía, son diversos los puertos españoles que comienzan a elaborar sus memorias de sostenibilidad.

Este elemento positivista desde la perspectiva del desarrollo sostenible permite avanzar en la búsqueda de objetivos más ambiciosos. Así, como elemento aislado, cada Memoria de la Autoridad Portuaria cumple una extraordinaria función. Sin embargo, a través de un replanteamiento de la batería de indicadores (creando una metodología común de cálculo para todos los puertos) y, sobre todo, de la creación de un índice sintético que permita la comparabilidad entre puertos; se podría avanzar en la definición de pautas de eficiencia y eficacia y en la gestión de políticas comunes. (González-Laxe, et al. 2016)

\subsection{Memoria de sostenibilidad de las Autoridades Portuarias}

Conscientes de la relevancia que posee la comunicación y la transparencia en lo referido a la gestión de los puertos de cara a la sociedad, ciertas Autoridades Portuarias iniciaron los trabajos para implementar una nueva herramienta de control que contribuyese a mejorar el posicionamiento estratégico de un puerto en sus tres dimensiones del desarrollo sostenible (ambiental, económica, y social). Dicha herramienta de control había de permitir un mejor análisis y una mayor comprensión del posicionamiento portuario respecto al medioambiente, así como contribuiría a desempeñar un rol más activo de cara a mejorar las 
interacciones y las repercusiones socio-económicas que conlleva respecto a las actividades económicas, la problemática urbana y el entorno geográfico.

La elaboración de dicho instrumento serviría para que tanto la organización portuaria como los grupos actores de stakeholders conozcan las imbricaciones de las políticas, los resultados de las medidas y la eficiencia de las acciones. De este modo, las AAPP deberían satisfacer las necesidades de los muy variados agentes/actores en torno a los diversos servicios y actividades. Por ello, se trataría de aplicar un enfoque que logre un triple beneficio: alcanzar los objetivos financieros, satisfacer las necesidades de los stakeholders; y contribuir a la mejora del medio ambiente (Crespo et al., 2010). La referencia a los agentes de la comunidad portuaria resulta cada vez más obligada en la medida que "los stakeholders son considerados como aquellas entidades o individuos a los que pueden afectar de manera significativa las actividades, productos y/o servicios del entorno portuario; y cuyas acciones pueden afectar, dentro de lo razonable, a la capacidad de la organización para desarrollar con éxito sus estrategias y alcanzar los objetivos" (Giner el al.,2011).

La mayor imbricación de las AAPP ha hecho que algunas de ellas hayan liderado esta dinámica; y hayan generado texto y propuestas de cara a su inmediata implementación. A modo de ejemplo, se puede citar el trabajo de las AAPP de Valencia y A Coruña, que siguieron los postulados del Global Reporting Initiative (GRI), del sistema de indicadores ambientales para el sistema portuario español (INDAPOR), y del Environmental Perfomance Indicators (EPI).

De esta forma, las memorias de sostenibilidad reflejan cuatro características específicas. La primera, ofrece una serie de pautas y recomendaciones concretas con objeto de ayudar a adoptar al sector portuario a los estándares de referencia; la segunda, facilita unas herramientas que permitan comunicar los resultados del comportamiento ambiental, económico y social; la tercera, permite reflexionar sobre los impactos económicos, ambientales y sociales derivados de las propias actividades que puedan afectar a los grupos de interés; y al mismo tiempo, coadyuvar a definir objetivos y compromisos relacionados con el desarrollo sostenible; y la cuarta, ofrece una batería de indicadores.

La presentación y el encuadre de los mismos siguiendo un esquema desagregado de los indicadores y sub-indicadores, revela un análisis complejo y multidimensional al recoger un amplio número de variables y de rasgos propios de la actividad principal y asociadas a la misma.

Los indicadores propuestos han de contar, en consecuencia, con una batería de variables relevantes por las que las AAPP puedan responder, con la opinión de los stakeholders relacionados y ante la presión de los ciudadanos, a los retos incluidos en las dimensiones económica, social, medio-ambiental e institucional; $\mathrm{y}$, al mismo tiempo, poder garantizar la coherencia de la memoria y la armonización de los datos/variables en todo el Sistema Portuario Español. Son, sin duda, un paso adelante muy significativo tanto en su apuesta por la eficiencia como en sus posibilidades de benchmarking.

\section{2. Índice sintético de sostenibilidad para puertos}

Una segunda línea de análisis consistió en diseñar un índice sintético global de sostenibilidad para implementar en los puertos españoles; el cual, aún dentro de su complejidad, pueda aplicarse a distintos ámbitos y esté construido en base a una metodología contrastada y con posibilidades de desarrollo. 
La originalidad de la propuesta de estudio radica en el papel que desempeñan los indicadores de sostenibilidad. La construcción de un índice global capaz de definir tipologías y situaciones diferenciadas, tanto para ser objeto de una evaluación permanente como de análisis de benchmarking, resulta extraordinariamente útil y necesaria. La combinación de un amplio panel de indicadores, representativos y explicativos del funcionamiento de una actividad permite, no solamente diagnosticar los niveles de cumplimiento de los objetivos prioritarios, sino también conocer con exactitud los grados de realización de las tareas encomendadas fijadas en los programas o ejes definidos. Asimismo, al construir un panel de indicadores homologables (y consiguientemente testables) se puede aplicar a localizaciones específicas y permite, igualmente, la comparabilidad entre situaciones diferenciadas.

La utilidad de este indicador global queda reflejada en que resulta básico para los futuros planes de empresas en lo que atañe a las apuestas de desarrollo sostenible y, de la misma forma, permite enfocar con mayor racionalidad las propuestas de futuro. Es de destacar su adecuación al diagnóstico de la realidad portuaria, lo que implica (como puede fácilmente inferirse) que su análisis permite abarcar ámbitos más extensos y amplios que la propia medición del impacto ambiental de las actividades de los puertos, a la vez que coadyuva a plantear nuevas estrategias en lo referente a la sostenibilidad (González-Laxe et al. 2016, 2017).

Bajo los presupuestos anteriores, se ha formulado y definido un denominado Índice Sintético de Sostenibilidad de los Puertos que se estructura de forma piramidal. De esta forma, el índice sintético se conforma a su vez de cuatro componentes o dimensiones divididas en diversos indicadores. Éstos provienen del cálculo de un determinado número de sub-indicadores, compuestos - cada uno de ellos- por una serie de variables. Dichas variables se definen a través de las contenidas en la Guía para la elaboración de Memorias de Sostenibilidad del Sistema Portuario Español y aquellas otras que se consideren indispensables para le generación del índice sintético. Las variables objeto de selección deben agruparse en sub-indicadores que, a su vez, se concentran en indicadores. Por último, los indicadores así agrupados se distribuyen en las cuatro conocidas dimensiones de la sostenibilidad a analizar: económica, institucional, medioambiental y social, los cuáles a su vez, compondrán el Índice Sintético. (ver tabla nº 3). 
Tabla $\mathbf{n}^{\mathbf{0}}$ 3. Composición del Índice Sintético: dimensiones, indicadores, sub-indicadores y número de variables.

\begin{tabular}{|c|c|c|c|}
\hline DIMENSIÓN & INDICADOR & SUB-INDICADOR & $\begin{array}{ll}\text { NÚMERO DE } \\
\text { VARIABLES }\end{array}$ \\
\hline \multirow{10}{*}{ Económica } & \multirow{5}{*}{ Estructura Económica } & Valor Económico Generado y Distribuido & 4 \\
\hline & & Situación Económica y Financiera & 4 \\
\hline & & Nivel y Estructura de las Inversiones & 3 \\
\hline & & Subvenciones Recibidas & 1 \\
\hline & & Retribución Capital Humano & 1 \\
\hline & \multirow{3}{*}{$\begin{array}{l}\text { Impacto Económico - } \\
\text { Social }\end{array}$} & Programa Beneficios Sociales & 1 \\
\hline & & Impuestos y Cargas Sociales & 1 \\
\hline & & Indicador Impacto Económico & 2 \\
\hline & \multirow{2}{*}{ Negocio y Servicios } & Servicios & 3 \\
\hline & & Negocios & 4 \\
\hline \multirow{6}{*}{ Institucional } & \multirow{4}{*}{ Capacidad Institucional } & Gastos I+D+i y Otros & 1 \\
\hline & & Proyección social & 1 \\
\hline & & Cumplimiento Normativo & 1 \\
\hline & & Infraestructuras y Servicios & 5 \\
\hline & \multirow{2}{*}{$\begin{array}{l}\text { Protección Capital } \\
\text { Humano y Natural }\end{array}$} & Riesgos para Capital Humano & 2 \\
\hline & & Riesgos para Capital Natural & 1 \\
\hline \multirow{13}{*}{ Medioambiental } & \multirow{4}{*}{$\begin{array}{l}\text { Gestión } \\
\text { Medioambiental }\end{array}$} & $\begin{array}{l}\text { Comportamiento Económico de la AP en } \\
\text { Medioambiente }\end{array}$ & 2 \\
\hline & & Sistemas de gestión & 1 \\
\hline & & Accidentes Medioambientales & 1 \\
\hline & & Formación ambiental & 1 \\
\hline & \multirow{4}{*}{ Ecoeficiencia } & Consumo de Materiales de Construcción & 1 \\
\hline & & Consumo Fuentes de Energía & 2 \\
\hline & & Consumo de Agua & 1 \\
\hline & & Energías Renovables & 1 \\
\hline & \multirow{4}{*}{ Calidad Ambiental } & \begin{tabular}{lrrrr|} 
Calidad del & Aire & y & Emisiones \\
Contaminantes & & & \\
\end{tabular} & 1 \\
\hline & & Calidad de las Aguas Interiores (zona I) & 1 \\
\hline & & \begin{tabular}{llllll|}
$\begin{array}{l}\text { Calidad del } \\
\text { Subterráneas }\end{array}$ & Suelo y & de & las & Aguas \\
\end{tabular} & 1 \\
\hline & & Producción y Gestión Residuos & 2 \\
\hline & Biodiversidad & Dragados & 2 \\
\hline \multirow{7}{*}{ Social } & Capital Social & Empleo & 3 \\
\hline & \multirow{2}{*}{ Capital Humano } & Nivel Educativo & 1 \\
\hline & & Formación & 1 \\
\hline & \multirow{2}{*}{ Equidad } & Igualdad de Género & 2 \\
\hline & & Estructura Laboral & 2 \\
\hline & \multirow{2}{*}{ Salud } & Siniestralidad Laboral & 2 \\
\hline & & Salud y Seguridad en el Trabajo & 1 \\
\hline 4 & 13 & 36 & 65 \\
\hline
\end{tabular}

Fuente: Elaboración propia. 


\section{Conclusiones.}

El desarrollo sostenible es un tema emergente en los actuales desarrollos portuarios; en la medida que cada vez son mayores los trabajos que tratan de exponer y medir la sostenibilidad de los puertos. La amplia literatura sobre la gestión portuaria se había concentrado en los análisis de eficiencia (Chang,2013) y en los de sensibilización medioambiental (Lam \& Notteboom,2014). O sea, los enfoques hasta el momento han priorizado las perspectivas medio-ambientales y económicas más que las dimensiones sociales e institucionales.

La mayoría de las administraciones portuarias todavía se encuentran acomodándose a las consecuencias de una mayor apertura global y de un aumento de los niveles de responsabilidad social colectiva. Los avances que se han llevado a cabo hasta el momento insisten en comunicar a la sociedad el comportamiento de ciertos parámetros económicos, medio-ambientales y sociales, incluidos en las memorias de gestión. Los objetivos se orientaron a mejorar y aumentar determinadas variables concernientes a los niveles de optimización relativos a la eficiencia de los tráficos, al posicionamiento del puerto, a la mejora de accesibilidad entre los modos de transporte, a la captación de inversiones, a asegurar un ambiente limpio y, finalmente, a resultar atractivo para el entorno y la ciudad. Más tarde, los análisis se han centrado en detallar los aspectos materiales, tales como: el transporte marítimo seguro y sustentable; la sustentabilidad medio-ambiental; la sustentabilidad financiera; la contribución económica; el apoyo internacional y el compromiso hacia la comunidad; y la actuación sobre la gente y cultura.

Finalmente, hay trabajos que tratan de enfocar la estrategia de compromiso siguiendo los criterios de la materialidad de los puertos mundiales subrayando, en este sentido, cinco aspectos: la performance medio-ambiental (impactos en las operaciones del puerto sobre los ecosistemas, industrias y comunidades que rodean al puerto); la conservación de las fuentes de energía y energías renovables; la solidez financiera y atracción de nuevos capitales y financiamiento; los recursos humanos y la promoción del cumplimiento de sus derechos básicos (salud, seguridad, capacitación); y el cumplimiento normativo y el desarrollo de las relaciones gubernamentales.

La complejidad de las Memorias y la inclusión de multitud de variables hacen que se aprecie una amplia diversidad de realidades y una discontinuidad de las actividades, dado el gran número de stakeholders que intervienen y que se relacionan con la actividad portuaria.

La sostenibilidad en el sistema portuario español se ha convertido en un elemento clave del desarrollo portuario, de acuerdo con los cambios legislativos introducidos en el año 2010. Aunque no todas las Autoridades Portuarias presentan de manera precisa sus Memorias de Sostenibilidad, una gran mayoría de las mismas efectúan tal ejercicio contribuyendo, con ello, a un mejor ejercicio de responsabilidad corporativa y a una mejora de las relaciones puerto-ciudad. La exigencia de dicha información y transparencia ha de constituir un nuevo desafío en los tiempos actuales.

\section{BIBLIOGRAFIA}

Acciaro, M.T., Vaneslander, C., Sys, C., Ferrari, A, Roumboutsos, G., Lam, J. et al. (2014). Environmental Sustainability in Seaports: A framework for Successful Innovation. Maritime Policy and Management. 41(5). 480-500. 
Chang, Y-T. (2013). Environmental Efficiency of Ports: A Data Envelopment Analysis Approach. Maritime Policy and Management. 40(5). 467-478.

Comisión Europea (1997). Libro Verde sobre los puertos y las infraestructuras marítimas. COM (1997) 678.

Crespo, C., Giner, A., \& Ripoll, V. (2010). Análisis de indicadores económicos en las memorias de sostenibilidad: el caso del sistema portuario español. Revista de sostenibilidad social de la empresa. 2 (1). 95-114.

Doerr. O. (2011). Políticas Portuarias Sostenibles. Boletín FAL, nº 299. CEPAL. Santiago de Chile.

Giner, A., Ripoll, V., Dickinson, Y., \& Orama, A. (2011). Evolución de la gestión de la información del sector portuario a través de la Memoria de Sostenibilidad como instrumento para la mejora de la información en el sector portuario. Revista de Contabilidade do Mestrado en Ciencias Contábeis. UERJ, 16(2) 67-78. Rio de Janeiro.

Giner, A., Silva, F., Lunkes, R.J., Mateo, V., \& Crespo, C. (2012). Sustentabilidade ambiental: Um estudo na Autoridade Portuaria de Valencia. Revista de Gestao, Finanzas e Contabilidade. UNEB. Salvador. 2 (1). 2-20.

González-Laxe, F., Martín-Bermúdez, F., Martin, F., \& Novo, I. (2016). Sustainability and the Spanish Ports System. Analysis of the relationship between economic and environmental indicators. Marine Pollution Bulletin. 113(1), 232-239.

González-Laxe, F., Martín-Bermúdez, F., Martin, F., \& Novo, I. (2017). Assessment of port sustainability through synthetic Index. Application to the Spanish case. Marine Pollution Bulletin. 119 (1), 220-225.

Kitzmann, D., \& Asmus, M. (2006). Gestao ambiental portuária: desafios e possiblidades. RAP. 40(6). 1041-1060.

Lam, J., \& Notteboom, Th. (2014). The greening of ports: a comparison of port management tools used by leading ports in Asia and Europe. Transports Reviews. 34(2). 169-189.

Papaefthimiou, S., Sitzimis, I., \& Andriosopoulos, K. (2017). A methodological approach for environmental characterization of ports. Maritime Policy and Management. 46(1). 81-93.

Peris-Mora, E., Diez, J.M., Subirats, A., Ibáñez, S., \& Alvarez, P. (2005). Development of a system of indicators for sustainable port management. Marine Pollution Bulletin. 50, 16491660.

\section{$\underline{\text { ANEXO }}$}

Desglose de los indicadores y sub-indicadores agregados según las cuatro dimensiones propuestas para un análisis empírico. 
Análisis de los indicadores y sub-indicadores agregados por dimensiones

\begin{tabular}{|c|c|c|c|}
\hline $\begin{array}{c}\text { DIMENSIÓN } \\
\text { INSTITUCIONAL }\end{array}$ & $\begin{array}{l}\text { DIMENSIÓN } \\
\text { ECONÓMICA }\end{array}$ & $\begin{array}{l}\text { DIMENSIÓN } \\
\text { SOCIAL }\end{array}$ & $\begin{array}{l}\text { DIMENSIÓN } \\
\text { AMBIENTAL }\end{array}$ \\
\hline $\begin{array}{c}\text { Infraestructuras y } \\
\text { capacidad }\end{array}$ & Rentabilidad: & Empleo: & $\begin{array}{l}\text { Sistemas de gestión } \\
\text { ambiental (SGA): }\end{array}$ \\
\hline $\begin{array}{l}\text { *Evolución superficie } \\
\text { terrestre. } \\
\text { *Trafico mercancías. } \\
\text { *Distribución de la superficie } \\
\text { terrestre y concesionada. } \\
\text { *Uso de muelles por tipo de } \\
\text { actividad. } \\
\text { *Número terminales por } \\
\text { tipología. } \\
\text { *Accesos terrestres: viario y } \\
\text { ferroviario. }\end{array}$ & $\begin{array}{l}\text { *Rentabilidad sobre } \\
\text { activos. } \\
\text { *Ebitda generado por } \\
\text { tonelada movida. } \\
\text { *Deuda. } \\
\text { *Gastos explotación } \\
\text { respecto a los ingresos. }\end{array}$ & $\begin{array}{l}\text { *Evolución } \\
\text { empleo. } \\
\text { *Distribución } \\
\text { empleo eventual } \\
\text { respecto al fijo. } \\
\text { *Distribución de la } \\
\text { plantilla por áreas } \\
\text { de actividad. } \\
\text { *Porcentaje } \\
\text { empleados } \\
\text { cubiertos por } \\
\text { convenios }\end{array}$ & $\begin{array}{l}\text { *Grado de } \\
\text { implantación de los } \\
\text { SGA. } \\
\text { *Gastos en } \\
\text { caracterización } \\
\text { ambiental. } \\
\text { *Gastos en limpieza de } \\
\text { superficie terrestres. } \\
\text { *Gastos en limpieza de } \\
\text { lámina de agua. }\end{array}$ \\
\hline $\begin{array}{l}\text { Generación de } \\
\text { infraestructuras: }\end{array}$ & $\begin{array}{l}\text { Estructura de las } \\
\text { inversiones: }\end{array}$ & Participación: & Calidad del aire: \\
\hline $\begin{array}{l}\text { *Inversión en infraestructuras } \\
\text { básicas y complementarias. } \\
\text { *Promoción industrial y } \\
\text { logística. }\end{array}$ & $\begin{array}{l}\text { *Inversión pública } \\
\text { respecto al cash-flow. } \\
\text { *Inversiones ajenas } \\
\text { respecto a las públicas. } \\
\text { *Inversiones públicas } \\
\text { respecto a los activos. }\end{array}$ & $\begin{array}{l}\text { *Número de } \\
\text { representantes } \\
\text { sindicales en el } \\
\text { Comité de } \\
\text { empresa. } \\
\text { *Organismos de } \\
\text { participación de los } \\
\text { trabajadores. }\end{array}$ & $\begin{array}{l}\text { *Porcentajes de quejas } \\
\text { por emisión a la } \\
\text { atmosfera. } \\
\text { *Medidas adoptadas } \\
\text { para minimizar } \\
\text { emisiones atmosféricas. }\end{array}$ \\
\hline $\begin{array}{l}\text { Transporte y movilidad } \\
\text { sostenible: }\end{array}$ & Negocios y Servicios: & Formación: & Calidad del agua: \\
\hline $\begin{array}{l}\text { *Inversión en intermodalidad, } \\
\text { *Coordinación entre modos } \\
\text { de transporte. } \\
\text { *Mercancías movidas por } \\
\text { FFCC. } \\
\text { *Mercancías movidas por } \\
\text { carretera+FFCC. } \\
\text { *Mercancías movidas por } \\
\text { Ro-Ro. }\end{array}$ & $\begin{array}{l}\text { *Ingresos por tasa de } \\
\text { ocupación respecto al } \\
\text { importe neto de cifra de } \\
\text { negocios. } \\
\text { *Ingresos por tasas de } \\
\text { de actividad respecto al } \\
\text { importe neto de cifra de } \\
\text { negocios. } \\
\text { *Rendimiento } \\
\text { superficie por } \\
\text { concesiones. } \\
\text { *Rendimiento de los } \\
\text { muelles activos. }\end{array}$ & $\begin{array}{l}\text { *Número de } \\
\text { programas } \\
\text { formativos. } \\
\text { *Porcentaje de } \\
\text { trabajadores que } \\
\text { siguen programas } \\
\text { de formación. } \\
\text { *Promedio de } \\
\text { horas de fación por } \\
\text { trabajador. }\end{array}$ & $\begin{array}{l}\text { *Principales focos de } \\
\text { vertidos al agua } \\
\text { portuaria. } \\
\text { *Medidas adaptadas } \\
\text { para mejora de la } \\
\text { calidad del agua. } \\
\text { *Porcentaje de } \\
\text { superficie de servicios } \\
\text { con red de aguas } \\
\text { residuales conectadas } \\
\text { al colector municipal o } \\
\text { EDAR. }\end{array}$ \\
\hline Tráficos: & Productividad: & Género: & Calidad acústica: \\
\hline $\begin{array}{l}\text { *Evolución mercancías. } \\
\text { *Distribución de los tipos de } \\
\text { tráficos. } \\
\text { *Tráficos mercancías según } \\
\text { import/export. } \\
\text { *Tráficos pasajeros. } \\
\text { *Pesca capturada. }\end{array}$ & $\begin{array}{l}\text { *Generación importe } \\
\text { neto de cifras de } \\
\text { negocios por empleado. } \\
\text { *Ratio Ebitda por } \\
\text { empleado }\end{array}$ & $\begin{array}{l}\text { *Porcentaje de } \\
\text { mujeres sobre el } \\
\text { total de empleados. }\end{array}$ & $\begin{array}{l}\text { *Principales focos de } \\
\text { emisiones acústicas. } \\
\text { *Porcentaje de quejas } \\
\text { por emisiones } \\
\text { acústicas. } \\
\text { *Medidas adoptadas } \\
\text { para mejora de la } \\
\text { calidad acústica. }\end{array}$ \\
\hline $\begin{array}{l}\text { Dinamizador del comercio y } \\
\text { competitividad: }\end{array}$ & & $\begin{array}{l}\text { Estructura } \\
\text { Plantilla: }\end{array}$ & Residuos: \\
\hline $\begin{array}{l}\text { *Distribución de tráficos } \\
\text { import/export/nacional. } \\
\text { *Distribución tráficos por }\end{array}$ & & $\begin{array}{l}\text { *Distribución de } \\
\text { los empleados } \\
\text { atendiendo a la }\end{array}$ & $\begin{array}{l}\text { *Principales fuentes de } \\
\text { residuos. } \\
\text { *Medidas adoptadas }\end{array}$ \\
\hline
\end{tabular}




\begin{tabular}{|c|c|c|}
\hline $\begin{array}{l}\text { áreas geográficas. } \\
\text { *Tráfico exportado según } \\
\text { países destino. } \\
\text { *Tráficos importados según } \\
\text { país origen. } \\
\text { *Sectores atendidos. }\end{array}$ & edad. & $\begin{array}{l}\text { para mejorar la } \\
\text { manipulación de } \\
\text { residuos. } \\
\text { *Clasificación de los } \\
\text { residuos. } \\
\text { *Gestión material } \\
\text { dragado. } \\
\text { * Residuos procedentes } \\
\text { de buques (MARPOL, } \\
\text { anexos I y V). }\end{array}$ \\
\hline Servicios: & $\begin{array}{l}\text { Seguridad y } \\
\text { Salud: }\end{array}$ & Biodiversidad: \\
\hline $\begin{array}{l}\text { *Distribución empresas } \\
\text { prestadoras servicios. } \\
\text { *Superficie concesionada. } \\
\text { *Distribución mercancías } \\
\text { movidas en terminales } \\
\text { concesionadas. }\end{array}$ & $\begin{array}{l}\text { *Implantación } \\
\text { sistema OHSAS } \\
\text { por servicios. } \\
\text { *Índice de } \\
\text { frecuencia por } \\
\text { accidentes. } \\
\text { *Índice de } \\
\text { gravedad de } \\
\text { accidentes. } \\
\text { *Índices de } \\
\text { absentismo }\end{array}$ & $\begin{array}{l}\text { *Especies protegidas } \\
\text { dentro y en las } \\
\text { proximidades del } \\
\text { puerto. }\end{array}$ \\
\hline $\begin{array}{l}\text { Transparencia y } \\
\text { concurrencia: }\end{array}$ & $\begin{array}{l}\text { Seguridad y } \\
\text { Protección: }\end{array}$ & Eco-eficiencia: \\
\hline $\begin{array}{l}\text { *Iniciativas para fomentar la } \\
\text { transparencia. }\end{array}$ & $\begin{array}{l}\text { *Número de } \\
\text { acciones } \\
\text { formativas de } \\
\text { carácter técnico en } \\
\text { relación a la } \\
\text { seguridad y } \\
\text { protección. } \\
\text { *Dispositivos de } \\
\text { ejercicio y } \\
\text { simulacros en } \\
\text { materia de } \\
\text { seguridad. } \\
\text { *Dispositivos de } \\
\text { ejercicios y } \\
\text { simulacros en } \\
\text { materia de } \\
\text { protección. }\end{array}$ & $\begin{array}{l}\text { *Consumo de agua. } \\
\text { *Consumo de } \\
\text { electricidad. } \\
\text { *Consumo de } \\
\text { combustibles. }\end{array}$ \\
\hline Calidad: & & $\begin{array}{l}\text { Gestión Ambiental de } \\
\text { la Comunidad } \\
\text { Portuaria: }\end{array}$ \\
\hline $\begin{array}{l}\text { *Mecanismos de } \\
\text { implantación para mejora de } \\
\text { la calidad servicios. } \\
\text { *Empresas que cuentan con } \\
\text { bonificación de calidad. } \\
\text { * Mecanismos de valorización } \\
\text { de la calidad de los servicios. }\end{array}$ & & $\begin{array}{l}\text { *Listado de } \\
\text { condiciones exigidas en } \\
\text { los instrumentos de } \\
\text { regulación. }\end{array}$ \\
\hline Promoción comercial: & & \\
\hline $\begin{array}{l}\text { * Gastos en promoción } \\
\text { comercial sobre gastos } \\
\text { explotación. } \\
\text { Tecnología información e } \\
\text { innovación: }\end{array}$ & & \\
\hline
\end{tabular}


*Uso tecnologías de información

Cooperación institucional con el entorno:

*Colaboración con entidades, escuelas, universidades,

fundaciones.

*Jornadas de formación.

*Evolución inversiones

puerto-ciudad.

*Inversiones en seguridad.

*Inversiones en

medioambiental.

Herramientas de gestión:

*Gestión calidad.

*Gestión riesgos laborales.

*Gestión ambiental.

*Estándares de excelencia

gestión.

Fuente) Elaboración propia. 